\title{
HOW CHANGING $D[[x]]$ CHANGES ITS QUOTIENT FIELD
}

\author{
BY \\ PHILIP B. SHELDON( $\left.{ }^{(}\right)$
}

\begin{abstract}
Let $D[[x]]$ be the ring of formal power series over the commutative integral domain $D$. It is shown that changing $D[[x]]$ to $D[[x / a]]$ changes (i.e. increases) the quotient field by an infinite transcendence degree over the original field whenever $\bigcap_{i=1}^{\infty} a^{i} D=0$. From this it follows that if $D_{1}$ and $D_{2}$ are two distinct rings between the integers and the rational numbers, with $D_{1}$ contained in $D_{2}$, then the change in the ring of coefficients from $D_{1}[[x]]$ to $D_{2}[[x]]$ again yields a change in the quotient fields by an infinite transcendence degree. More generally, it is shown that $D$ is completely integrally closed iff any increase in the ring of coefficients yields an increase in the quotient field of $D[[x]]$. Moreover, $D$ is a one-dimensional Prüfer domain iff any change in the ring of coefficients from one overring of $D$ to another overring of $D$ yields a change in the quotient field of the respective power series rings. Finally it is shown that many of the domain properties of interest are really properties of their divisibility groups, and some examples are constructed by first constructing the required divisibility groups.
\end{abstract}

1. Introduction. Let $D$ be a commutative integral domain (with identity) with quotient field $K$, and let $D[[x]]$ denote the ring of formal power series with coefficients in $D$. The quotient field of this latter ring, denoted $Q(D[[x]])$, “ought to be", by analogy with the ring of polynomials over $D$, the same as the quotient field of $K[[x]]$. However, Gilmer $\left[\mathrm{G}_{3}\right]$ has shown that this is very rarely the case; one of the necessary conditions for the equality of these two quotient fields is that $\bigcap_{i=1}^{\infty} a^{i} D \neq 0$ for all nonzero elements $a$ in $D$. It is shown here that whenever the above condition fails-i.e., when $\bigcap a^{i} D=0$ for some nonzero $a$ in $D$-then apparently rather small increases in the size of the power series ring cause rather wild increases in the size of the quotient field. The two most striking results are the following:

(1) If $\bigcap a^{i} D=0$, then $Q(D[[x / a]])$ has infinite transcendence degree over $Q(D[[x]])$. (Theorem 2.1.)

And as an immediate corollary:

(2) If $D_{1}$ and $D_{2}$ are two distinct domains between the integers and the rational

Presented to the Society, January 22, 1971; received by the editors July 21, 1970.

AMS 1970 subject classifications. Primary 13J05, 13G05; Secondary 13A05, 13F05, 13B20, 13A15.

Key words and phrases. Infinite transcendence degree, completely integrally closed domains, one-dimensional Prüfer domains, complete integral closure, valuation rings, value group, divisibility group, semivaluations, lattice-ordered divisibility groups, Bezoutian domain.

${ }^{1}$ ) This is part of the author's doctoral dissertation at the University of Wisconsin under the direction of Professor Lawrence S. Levy, to whom the author is very grateful for his help and guidance. 
numbers with $D_{1}$ contained in $D_{2}$, then $Q\left(D_{2}[[x]]\right)$ has infinite transcendence degree over $Q\left(D_{1}[[x]]\right)$. (Corollary 2.2.)

Result (2) suggests the following question: What conditions on $D$ will guarantee that any increase in the ring of coefficients will lead to an increase in the quotient field of the corresponding power series ring? This question is answered completely and the result then extended slightly to yield the following new characterizations of completely integrally closed domains and one-dimensional Prüfer domains:

(3) $D$ is completely integrally closed iff for any domain $D_{1}$ contained in $K$ and properly containing $D, Q\left(D_{1}[[x]]\right) \neq Q(D[[x]])$. (Theorem 3.4.)

(4) $D$ is a Prüfer domain with Krull dimension one iff for any two distinct domains $D_{1}$ and $D_{2}$ containing $D$ and contained in $K, Q\left(D_{1}[[x]]\right) \neq Q\left(D_{2}[[x]]\right)$. (Theorem 3.5.)

Finally, we present some examples of pairs of domains which answer questions raised about the equality of the quotient fields of their respective power series rings. It is shown that many of the properties of interest in all of these domains are properties of their divisibility groups. In fact, our final examples are constructed by first constructing lattice-ordered abelian groups with certain specified properties, and then using Jaffard's theorem to show the existence of domains with these groups as their divisibility groups.

2. Infinite transcendence degree of $Q(D[[x / a]])$ over $Q(D[[x]])$. We begin with the statement of the main result of this section.

2.1. TheOrem. Let $D$ be an integral domain and let a be a nonzero element of $D$. If $\bigcap_{i=1}^{\infty} a^{i} D=0$, then $Q(D[[x / a]])$ has an infinite transcendence degree over $Q(D[[x]])$.

This theorem yields a corollary which we state and prove at once.

2.2. Corollary. If $D_{1}$ and $D_{2}$ are two distinct subrings of the field of rational numbers with $D_{1}$ contained in $D_{2}$, then $Q\left(D_{2}[[x]]\right)$ has infinite transcendence degree over $Q\left(D_{1}[[x]]\right)$.

Proof. First, any ring $D_{1}$ between the integers and the rationals is known to be a PID. (For example, apply $\left[G_{1}\right.$, Theorem 22.1, part 3, p. 306].) Moreover, any ring $D_{2}$ between $D_{1}$ and its quotient field contains the inverse of a nonzero nonunit of $D_{1}$. (Apply the QR-property [G-O.] Alternately, pick an element $b / a$ in $D_{2}-D_{1}$, where $b$ and $a$ are relatively prime integers. Then there exist integers $y$ and $z$ such that $y(b / a)+z=(y b+z a) / a=1 / a \in D_{2}$.) Also unique factorization in $D_{1}$ implies that this nonunit $a$ of $D_{1}$ will satisfy $\cap a^{i} D_{1}=0$. Hence we know from the theorem that $Q\left(D_{1}[[x / a]]\right)$ has infinite transcendence degree over $Q\left(D_{1}[[x]]\right)$. But since $D_{1}$ is contained in $D_{2}$, and since $a$ is a unit of $D_{2}$, then clearly $D_{1}[[x / a]]$ is contained in $D_{2}[[x]]$. Since the same containment must hold between their quotient fields, and since the smaller field already has infinite transcendence degree over $Q\left(D_{1}[[x]]\right)$, the proof is complete. 
REMARK. This corollary holds more generally in the case where $D_{1}$ and $D_{2}$ are any two distinct domains with $D_{1}$ contained in $D_{2}$ and with $P_{1} D_{2}=D_{2}$ for some minimal prime ideal $P_{1}$ of $D_{1}$. (Apply $\left[\mathrm{O}_{1}\right.$, Corollary 1.4, p. 323], and then follow the final three sentences of the proof.)

Before we begin the actual proof of the theorem, we need to develop some terminology and some preliminary results.

2.3. Definition. For any power series $\boldsymbol{p}$ in $K[[x]]$, we define the support of $\boldsymbol{p}$, denoted supp ( $\boldsymbol{p})$, to be the set of all nonnegative integers $s$ for which $x^{s}$ has a nonzero coefficient in $p$.

The idea to be used in constructing an infinite transcendence basis of $Q(D[[x / a]])$ over $Q(D[[x]])$ was inspired by the very interesting paper by Rivet $[\mathrm{R}]$. He shows, among other things, that if $a$ is a nonzero nonunit in a discrete rank-one valuation ring $D$, then any power series of the form $\sum_{j \in J}(x / a)^{j}$ with certain "large gaps" in its support $J$ will necessarily not be in $Q(D[[x]])$. His large-gap criterion, however, is not sufficient to guarantee that the power series is transcendental over $Q(D[[x]])$. For example, the power series $p=\sum_{j \in J}(x / a)^{j}$, where $J=\{1,2,4,8, \ldots\}$, satisfies his requirements; but in case $D$ has characteristic 2 , it satisfies the algebraic equation $a p^{2}-a p+x=0$ over $D[[x]]$.

Our first step will be to consider a finite set of power series $\boldsymbol{b}_{0}, \boldsymbol{b}_{1}, \ldots, \boldsymbol{b}_{R}$ in $D[[x / a]]$ with the following "Special Large Gap Property" (SLGP):

For each integer $n>0$, there is an integer $L$ such that every power (SLGP) from $x^{L-n}$ through $x^{L+n}$ has a zero coefficient in each of the $\boldsymbol{b}_{r}$, except that the coefficient of $x^{L}$ in $b_{0}$ is $a^{-L}$.

This property is much easier to visualize in terms of the $(R+1) \times \infty$ matrix whose $(r, s)$ entry is the coefficient of $x^{s}$ in the power series $b_{(r-1)}$. Thus the SLGP means that, for each $n$, there exists an $(R+1) \times(2 n+1)$ submatrix of the following form:

$$
\begin{aligned}
& x^{L-n} \quad x^{L-1} \quad x^{L} x^{L+1} \quad x^{L+n} \\
& \begin{array}{c|cccccc}
\boldsymbol{b}_{0} & 0, \ldots, & 0, & a^{-L}, & 0, \ldots, & 0 \\
\boldsymbol{b}_{1} & 0, \ldots, & 0, & 0, & 0, \ldots, & 0 \\
& \vdots & \vdots & \vdots & \vdots & & \vdots \\
\boldsymbol{b}_{R} & 0, \ldots, & 0, & 0, & 0, \ldots, & 0
\end{array} \mid
\end{aligned}
$$

2.4. Lemma. Let $D$ be a domain, and let a be a nonzero element of $D$. Let $\boldsymbol{b}_{0}, \boldsymbol{b}_{1}, \ldots, \boldsymbol{b}_{R}$ be a set of power series in $D[[x / a]]$ satisfying SLGP. Suppose there is a linear dependence relation,

$$
\boldsymbol{d}_{0} b_{0}+d_{1} b_{1}+\cdots+d_{R} b_{R}=0
$$

in which the coefficients $\boldsymbol{d}_{0}, \boldsymbol{d}_{1}, \ldots, \boldsymbol{d}_{R}$ are in $D[[x]]$, and $\boldsymbol{d}_{0} \neq 0$. Then $\bigcap a^{i} D \neq 0$.

Proof. Assume the hypotheses of the lemma are satisfied. Since we know $\boldsymbol{d}_{0} \neq 0$, we will denote by $k$ the index of its first nonzero coefficient $d_{0, k}$. Pick any $n$ greater than $k$. Let $L$ be the integer specified in the SLGP which corresponds to the value $n$. 
For each $r$ from 0 to $R$, define two new power series as follows:

$\boldsymbol{b}_{r}^{\prime}=$ the sum of the terms of $\boldsymbol{b}_{r}$ with degree less than $L-n$.

$\boldsymbol{b}_{r}^{\prime \prime}=$ the sum of the terms of $\boldsymbol{b}_{r}$ with degree greater than $L+n$.

Since the intervening terms are all zero, with one exception, we may rewrite the original $b_{r}$ thus:

$$
\begin{array}{cc}
b_{0}=b_{0}^{\prime}+a^{-L} x^{L}+b_{0}^{\prime \prime} \\
b_{1}=b_{1}^{\prime}+ \\
\vdots \quad \vdots \quad \vdots & b_{1}^{\prime \prime} \\
b_{R} & =b_{R}^{\prime}+
\end{array}
$$

If we define two new power series $c^{\prime}$ and $c^{\prime \prime}$ to be

$$
\begin{aligned}
& c^{\prime}=d_{0} b_{0}^{\prime}+d_{1} b_{1}^{\prime}+\cdots+d_{R} b_{R}^{\prime}, \\
& c^{\prime \prime}=d_{0} b_{0}^{\prime \prime}+d_{1} b_{1}^{\prime \prime}+\cdots+d_{R} b_{R}^{\prime \prime},
\end{aligned}
$$

then the dependence relation (2.4.1) becomes

$$
c^{\prime}+d_{0}\left(a^{-L} x^{L}\right)+c^{\prime \prime}=0 .
$$

Observe that each of the power series $\boldsymbol{b}_{r}^{\prime \prime}$ has all zero coefficients before the $(L+n)$ thdegree term, and thence the same can be said for $c^{\prime \prime}$.

Recall that $\boldsymbol{b}_{0}^{\prime}, \boldsymbol{b}_{1}^{\prime}, \ldots, \boldsymbol{b}_{R}^{\prime}$ are all in $D[[x / a]]$, and all have no terms after the $(L-n)$ th-degree term. Thus if we multiply each one by $a^{L-n}$, it will become a power series in $D[[x]]$. Hence $\left(a^{L-n}\right) c^{\prime}$ is also in $D[[x]]$. If we multiply the equation (2.4.2) by $a^{L-n}$, we get

$$
a^{L-n} c^{\prime}+d_{0}\left(a^{-n} x^{L}\right)+a^{L-n} c^{\prime \prime}=0 .
$$

Now consider the total coefficient of $x^{L+k}$ in the left-hand expression of (2.4.3). This coefficient must be zero, since the whole expression is zero. On the other hand, it must be equal to the sum of the coefficients of $x^{L+k}$ in each of the three terms in the expression. The last term has a zero coefficient, since $L+k<L+n$, and since there are only zeroes before the $(L+n)$ th-degree term. The first term is a power series in $D[[x]]$, so it has a coefficient in $D$. So we are forced to conclude that the middle term has its coefficient of $x^{L+k}$ in $D$ as well. But this coefficient is precisely $d_{0, k} a^{-n}$. Hence $d_{0, k} \in a^{n} D$. But our original choice of $n$ could have been replaced by any larger value, and still lead to the same conclusion. Hence

$$
0 \neq d_{0, k} \in \bigcap_{n=1}^{\infty} a^{n} D .
$$

This completes the proof of Lemma 2.4 .

Proof of Theorem 2.1. Assume $a$ is a nonzero element of $D$ which satisfies $\bigcap_{i=1}^{\infty} a^{i} D=0$. Let $f$ be a function from the positive integers into the positive integers with the following properties:

(a) $f(i)>i, i=1,2, \ldots$,

(b) $f(i+1)>f(i)+1, i=1,2, \ldots$,

(c) for each $N>0$, there exists an $I>0$ such that $i \geqq I$ implies $f(i+1)>N \cdot f(i)$. 
An example of such a function is $f(i)=2^{2^{1}}$. Let $f^{2}$ denote $f \circ f$, and so forth.

Now we define an infinite sequence of power series in $D[[x / a]]$ as follows:

$$
\begin{gathered}
a_{1}=\sum_{i=1}^{\infty}(x / a)^{f(i)} \\
a_{2}=\sum_{i=1}^{\infty}(x / a)^{f^{2}(i)} \\
\vdots \\
a_{j}=\sum_{i=1}^{\infty}(x / a)^{f^{\prime}(i)}
\end{gathered}
$$

Note that $\operatorname{supp}\left(a_{1}\right)=\operatorname{image}(f)$, and $\operatorname{supp}\left(a_{2}\right)=\operatorname{im}\left(f^{2}\right) \subset \operatorname{im}(f)=\operatorname{supp}\left(a_{1}\right)$ (where $\subset$ denotes proper inclusion). In general, $\operatorname{supp}\left(a_{j+1}\right) \subset \operatorname{supp}\left(a_{j}\right)$. Also note that, by our choice of $f, a_{1}$ has very large gaps in its support, and each successive $a_{j}$ has increasingly larger gaps, since its support is a very small subset of the support of the preceding power series.

Now suppose the set $\left\{\boldsymbol{a}_{j}\right\}$ is algebraically dependent over $Q(D[[x]])$. Then there is an integer $J$ and a polynomial $P\left(t_{1}, \ldots, t_{J}\right)$ with coefficients in $D[[x]]$ (we can always multiply through by a common denominator from $D[[x]]$, if the original coefficients are fractions of elements of $D[[x]])$ which when evaluated at $a_{1}, a_{2}, \ldots$, $a_{J}$ gives the zero power series.

Consider the set $M$ of all power series of the form $a_{1}^{e(1)} \cdots a_{J}^{e(J)}$ corresponding to those monomial expressions $t_{1}^{e(1)} \cdots t_{J}^{e(J)}$ which occur in $P$ with nonzero coefficient. We shall show that this set satisfies the hypothesis of Lemma 2.4, i.e. the Special Large Gap Property. Thus the equation of algebraic dependence, $P\left(a_{1}, \ldots, a_{J}\right)=0$, is actually an equation of linear dependence among the elements of $M$ (with all coefficients nonzero), and we may conclude that $\cap a^{i} D \neq 0$. Since the assumption that the set $\left\{\boldsymbol{a}_{j}\right\}$ is algebraically dependent will thus lead to a contradiction of our choice of $a$, we must conclude that the set is algebraically independent, and the theorem will then be proved.

All that remains is to demonstrate the Special Large Gaps in the set $M$. First we need a lemma.

2.5. Lemma. Let $p_{1}, \ldots, p_{Q}$ be power series in $K[[x]]$, and let $S$ be a positive integer. For each $q$, let $s^{\prime}(q)$ denote the largest element of $\operatorname{supp}\left(\boldsymbol{p}_{q}\right)$ which is less than $S$, and let $s^{\prime \prime}(q)$ denote the second largest element. In other words, we may rewrite each $\boldsymbol{p}_{q}$ as follows:

$$
\boldsymbol{p}_{q}=\cdots+p_{q, s^{\prime \prime}(q)} x^{x^{\prime \prime}(q)}+0+\cdots+0+p_{q, s^{\prime}(q)} x^{s^{\prime}(q)}+0+\cdots+0+p_{q, S} x^{S}+\cdots
$$

Let $S^{\prime}$ denote $s^{\prime}(1)+\cdots+s^{\prime}(Q)$. Then

(A) If $S^{\prime}<S$, it follows that $S^{\prime}$ is the largest element of $\operatorname{supp}\left(\prod_{q=1}^{Q} p_{q}\right)$ which is less than $S$ and the coefficient of the term $x^{S^{\prime}}$ in $\prod p_{q}$ is precisely $\prod_{q=1}^{Q} p_{q, s^{\prime}(q)}$.

(B) If, in addition to the above conditions, we have $s^{\prime}(q)-s^{\prime \prime}(q)$ always greater than some integer $n$, then the largest element of supp $\left(\prod p_{q}\right)$ which is less than $S^{\prime}$ 
will also be less than $S^{\prime}-n$. (Note that we make no attempt to specify the coefficient of this term.)

The proof of this lemma is a straightforward application of the definition of multiplication of power series, and we omit the details.

We shall apply this lemma to sets chosen from $a_{1}, \ldots, a_{J}$, permitting repetitions. Thus we will establish the existence of gaps in certain of the products of these sets of power series, namely the monomial expressions that occur in the set $M$, and thereby prove that $M$ has the SLGP. We shall shortly specify which element of $M$ is to play the special role of $\boldsymbol{b}_{0}$ in this set, and then demonstrate the existence of the required $L$ for each given $n$.

For any choice of an element $S$ common to the supports of $\boldsymbol{a}_{1}$ up to $\boldsymbol{a}_{J}$, we can define $s^{\prime}(j)$ and $s^{\prime \prime}(j)$ as in the lemma to be the largest and second largest elements of supp $\left(a_{j}\right)$ which are smaller than $S$ (assuming that there are at least two elements of supp $\left(a_{j}\right)$ which are smaller than $S$ ). An illustration of such a choice is given in the diagram below, in which slashes on the various lines correspond to integers in the supports of $a_{1}, a_{2}$, and $a_{3}$.

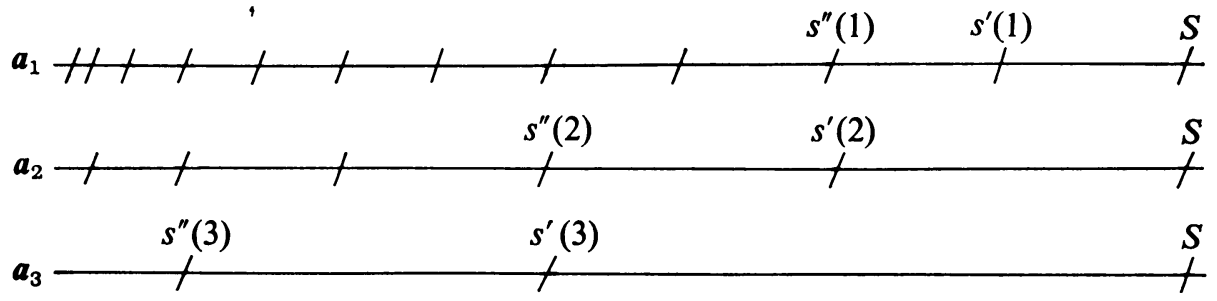

We claim that, for any choice of $n$ and $N$, by choosing $S$ large enough we can guarantee the following five conditions:

(I) $s^{\prime}(J)<s^{\prime}(J-1)<\cdots<s^{\prime}(1)$.

(II) $N \cdot s^{\prime}(j)<s^{\prime}(j-1)$, for $j=2, \ldots, J$.

(III) $N \cdot s^{\prime}(1)<S$.

(IV) $s^{\prime}(j)>n$, for $j=1, \ldots, J$.

(V) $s^{\prime}(j)-s^{\prime \prime}(j)>n$, for $j=1, \ldots, J$.

The proof of this claim will follow directly from the following observation. For any two elements that are adjacent in supp $\left(a_{j}\right)$ (i.e. no integer between them also lies in supp $\left(\boldsymbol{a}_{j}\right)$ ), they cannot both lie in the support of $\boldsymbol{a}_{j+1}$. To see this, suppose $s_{1}$ and $s_{2}$ are adjacent elements in supp $\left(a_{j}\right)$. Then by the definition of $a_{j}$, there is an integer $i$ such that $s_{1}=f^{j}(i)$ and $s_{2}=f^{j}(i+1)$. If they also both lie in supp $\left(a_{j+1}\right)$, they are necessarily still adjacent, since $\operatorname{supp}\left(a_{j+1}\right)$ is a subset of $\operatorname{supp}\left(\boldsymbol{a}_{j}\right)$. Hence there is an integer $i^{\prime}$ satisfying $s_{1}=f^{j+1}\left(i^{\prime}\right)$ and $s_{2}=f^{j+1}\left(i^{\prime}+1\right)$. Equating the two expressions for $s_{1}$ and the two for $s_{2}$, and using the fact that $f^{j}$ is one-to-one, we see that $f\left(i^{\prime}\right)=i$ and $f\left(i^{\prime}+1\right)=i+1$. This contradicts our choice of $f$, since $f\left(i^{\prime}+1\right)$ $>f\left(i^{\prime}\right)+1$ for any integer $i^{\prime}$, and thus the original observation is verified.

Now, given $n$ and $N$, we will specify how to choose $S$ to satisfy conditions (I) to (V). First, in supp $\left(a_{1}\right)$ find an entry greater than $n$. Next, go out further in $\operatorname{supp}\left(a_{1}\right)$, 
if need be, to the point where $N$ times each element is less than the following one. In particular, since we may take $N$ to be at least two, this will guarantee that the gaps from here on are all of length greater than $n$. Now pick the next entry in $\operatorname{supp}\left(a_{1}\right)$ which is also in supp $\left(a_{J}\right)$, hence in all the intervening ones as well. This entry will serve as $s^{\prime \prime}(J)$, the following element in $\operatorname{supp}\left(\boldsymbol{a}_{J}\right)$ will be $s^{\prime}(J)$, and the next one will be the required $S$.

To see that (I) holds, note that by definition $s^{\prime}(j)$ and $S$ are adjacent in supp $\left(a_{j}\right)$. Since $S$ is in supp $\left(a_{j+1}\right)$, we see that $s^{\prime}(j)$ cannot be in there, nor can any number between it and $S$. Hence the largest entry in supp $\left(a_{j+1}\right)$ which is less than $S$ is necessarily less than $s^{\prime}(j)$ as well. In other words, $s^{\prime}(j+1)<s^{\prime}(j)$.

Next, (II) and (III) both follow from the fact that all of the $s^{\prime}$-values being considered are in supp $\left(a_{1}\right)$ and, furthermore, are out beyond the point where $N$ times any entry is less than any subsequent entry. But (I) shows that $s^{\prime}(j-1)$ is always a later entry than $s^{\prime}(j)$; and of course, $S$ is a later entry than $s^{\prime}(1)$, by the definition of $s^{\prime}(1)$. This is just what we need to prove (II) and (III).

Finally, since all of the values being considered lie out beyond $n$, and beyond the point where the gaps are all greater than $n,(\mathrm{IV})$ and $(\mathrm{V})$ are reduced to trivialities.

Now let $T$ be the total degree of $P$, that is, the largest exponent-sum among all of the monomials in $P$ with nonzero coefficients. Let $N=T+1$. Let $n$ denote an arbitrary choice of a positive integer, the one we need to verify the SLGP. Finally let $S$ be the value as in the preceding claims which assures that conditions (I) to (V) are satisfied for these particular values of $n$ and $N$.

Order the elements of $M$ lexicographically, using the exponents of $a_{1}$, then $a_{2}$, and so forth, to establish the ordering. Denote the largest term in this ordering by

$$
\boldsymbol{m}^{\prime}=a_{1}^{e^{\prime}(1)} \cdots \boldsymbol{a}_{J}^{e^{\prime}(J)}
$$

(this element will play the role of $b_{0}$ in the SLGP), and let

$$
\boldsymbol{m}^{\prime \prime}=\boldsymbol{a}_{1}^{e^{\prime \prime}(1)} \cdots \boldsymbol{a}_{J}^{e^{\prime \prime}(J)}
$$

denote any other element of $M$.

Next, applying the results of Lemma 2.5 to $\boldsymbol{m}^{\prime}$ as a product of power series, we can easily see that the largest term in supp $\left(\boldsymbol{m}^{\prime}\right)$ which is less than $S$ is precisely $\sum_{j=1}^{J} e^{\prime}(j) s^{\prime}(j)$, provided only that this sum is less than $S$. But (calling this sum $L$ in anticipation of its role in the SLGP)

$$
\begin{aligned}
L & =\sum\left(e^{\prime}(j) s^{\prime}(j)\right) \\
& \leqq \sum\left(e^{\prime}(j) s^{\prime}(1)\right), \text { by }(\mathrm{I}), \\
& =\left(\sum e^{\prime}(j)\right) s^{\prime}(1) \\
& \leqq T \cdot s^{\prime}(1), \text { by definition of } T, \\
& =(N-1) s^{\prime}(1), \quad \text { by definition of } N, \\
& <S-s^{\prime}(1), \quad \text { by (III), } \\
& <S .
\end{aligned}
$$


This proves that the lemma may be applied, and, furthermore, shows that $L<S$ $-s^{\prime}(1)$. Since we also know by condition (IV) that $s^{\prime}(1)>n$, we see that $L+n<S$. Thus all coefficients of $\boldsymbol{m}^{\prime}$ from the $(L+1)$ th-degree term through the $(L+n)$ thdegree term are zero. To show the same gap occurs from $L-n$ through $L-1$, we consider the gaps $s^{\prime}(j)-s^{\prime \prime}(j)$, which we already know are always greater than $n$, by condition (V). Hence, by Lemma 2.5 , the second largest term in supp ( $\left.\boldsymbol{m}^{\prime}\right)$ which is less than $S$ is necessarily less than $L-n$. Finally, recall that in each power series $a_{j}$, the coefficient of the term $x^{s^{\prime}(j)}$ is always $a^{-s^{\prime}(j)}$. Hence the coefficient of the term $x^{L}$ in $m^{\prime}$, again by Lemma 2.5 , is $\prod_{j=1}^{J}\left(a^{-s^{\prime}(j)}\right)^{e^{\prime}(j)}$ which equals $a^{-L}$. This completes the demonstration that the monomial expression $\boldsymbol{m}^{\prime}$ satisfies the conditions required of $b_{0}$ in the SLGP. In other words, we have shown that we have constructed the first row

$$
\left(0, \ldots, 0, a^{-L}, 0, \ldots, 0\right)
$$

of the matrix as required.

To show that we can construct the remainder of the matrix, we need only show that all of the other rows are zero rows. That is, we must show that for our arbitrary element $\boldsymbol{m}^{\prime \prime}$ the largest element of supp $\left(\boldsymbol{m}^{\prime \prime}\right)$ which is less than $S$ is also less than $L-n$. This will insure that there are all zero terms in $m^{\prime \prime}$ from $x^{L-n}$ up to $x^{S}$, and necessarily all zeros from $x^{L-n}$ through $x^{L+n}$.

The same argument used for $\boldsymbol{m}^{\prime}$ shows that this largest element of $\operatorname{supp}\left(\boldsymbol{m}^{\prime \prime}\right)$ less than $S$ is $\sum_{j=1}^{J} e^{\prime \prime}(j) s^{\prime}(j)$. So we will be done if we can show that

$$
\sum e^{\prime}(j) s^{\prime}(j)-\sum e^{\prime \prime}(j) s^{\prime}(j)>n .
$$

Starting with $j=1$, we compare the exponents $e^{\prime}(j)$ and $e^{\prime \prime}(j)$. By definition of the lexicographic ordering, at the first time that they differ, say at $j_{0}, e^{\prime}\left(j_{0}\right)$ will be greater than $e^{\prime \prime}\left(j_{0}\right)$. We may ignore terms before $j_{0}$, since they all sum to zero, and try to prove

$$
\sum_{j=j_{0}}^{J} e^{\prime}(j) s^{\prime}(j)-\sum_{j=j_{0}}^{J} e^{\prime \prime}(j) s^{\prime}(j)>n .
$$

Rewriting this as

$$
\left[e^{\prime}\left(j_{0}\right)-e^{\prime \prime}\left(j_{0}\right)\right] s^{\prime}\left(j_{0}\right)+\sum_{j=j_{0}+1}^{J}\left(\left[e^{\prime}(j)-e^{\prime \prime}(j)\right] s^{\prime}(j)\right)>n,
$$

with $e^{\prime}\left(j_{0}\right)-e^{\prime \prime}\left(j_{0}\right) \geqq 1$, it will suffice to prove

$$
s^{\prime}\left(j_{0}\right)>n+\sum_{j=j_{0}+1}^{J}\left[e^{\prime \prime}(j)-e^{\prime}(j)\right] s^{\prime}(j) .
$$

Since $e^{\prime}(j)$ is nonnegative, we may ignore it and prove

$$
s^{\prime}\left(j_{0}\right)>n+\sum_{j=j_{0}+1}^{J} e^{\prime \prime}(j) s^{\prime}(j) .
$$


Since $e^{\prime \prime}(j)$ is also nonnegative, and since $s^{\prime}\left(j_{0}+1\right)>s^{\prime}\left(j_{0}+2\right)>\cdots$, we may replace each $s^{\prime}(j)$ by a larger value, and try to prove

$$
s^{\prime}\left(j_{0}\right)>n+\sum_{j=j_{0}+1}^{J} e^{\prime \prime}(j) s^{\prime}\left(j_{0}+1\right)=n+\left[\sum_{j=j_{0}+1}^{J} e^{\prime \prime}(j)\right] s^{\prime}\left(j_{0}+1\right) .
$$

But $\sum_{j=j_{0}+1}^{J} e^{\prime \prime}(j) \leqq T$, by definition of the total degree of $P$, so we need only consider

$$
s^{\prime}\left(j_{0}\right)>n+T \cdot s^{\prime}\left(j_{0}+1\right)
$$

But $n<s^{\prime}\left(j_{0}+1\right)$, so we may reduce the inequality to be proved to this:

$$
s^{\prime}\left(j_{0}\right)>s^{\prime}\left(j_{0}+1\right)+T \cdot s^{\prime}\left(j_{0}+1\right)=N \cdot s^{\prime}\left(j_{0}+1\right) .
$$

But from condition (II), we know our choice of $S$ gave us $s^{\prime}\left(j_{0}\right)>N \cdot s^{\prime}\left(j_{0}+1\right)$, and therefore we are done.

This completes the proof that the set $M$ satisfies the SLGP, and so completes the proof of Theorem 2.1 .

3. Relations between $Q\left(D_{1}[[x]]\right)$ and $Q\left(D_{2}[[x]]\right)$.

3.0. Preliminaries and notation. A good general reference for most of the concepts and terminology to be used in this and the following section is Robert Gilmer's book Multiplicative ideal theory $\left[\mathrm{G}_{1}\right]$.

Throughout this section, $D, D_{1}$, and $D_{2}$ will represent domains (with identity) which all have the same quotient field $K$. We will denote the sets of nonzero elements of $D, D_{1}$, and $D_{2}$ by $D^{*}, D_{1}^{*}$, and $D_{2}^{*}$ respectively. By an overring of $D$ we will mean a subring of $K$ containing $D$ (possibly equal to $D$ ), and a quotient overring of $D$ is an overring of form $D_{S}$, where $S$ is a multiplicative subset of $D^{*}$. In case $S$ is the complement of a prime ideal $P$ in $D$, the quotient ring $D_{S}$ is a local ring (i.e., it has a unique maximal ideal) and we call $D_{S}$ the localization of $D$ at $P$ and denote it $D_{P}$.

An element $k \in K$ is said to be almost integral over $D$ (also called quasi-integral in the literature) if there is a $d \in D^{*}$ such that for each $i, d k^{i} \in D . D$ is said to be completely integrally closed (c.i.c.) if no elements outside of $D$ are almost integral over $D$. The complete integral closure of $D$, denoted CIC $(D)$, is defined to be $\{k \in K \mid k$ is almost integral over $D\}$. It is well known that this set is an overring of $D$ which is always integrally closed, but not necessarily completely integrally closed $\left[G_{1}, \S 12\right]$.

The domain $D$ is said to be root closed (in $K$ ) if every element of $K$ which is a root of an element of $D$ is already in $D$.

By the dimension of a domain $D$ we will mean the Krull dimension of $D$, i.e., the length of the longest chain of proper prime ideals of $D$. For a valuation ring, the terms rank and dimension will be used interchangeably. In particular, a domain is one-dimensional if all proper prime ideals are maximal, and a valuation ring has rank one if it has just one proper prime ideal. 
Now we are ready to give our first indication of the relationship between the seemingly unrelated concepts of complete integral closure and quotient fields of power series. The foliowing lemma serves this purpose.

3.1. Lemma. Given the domain $D$ and an element $k \in K^{*}$, consider the following three conditions on them:

(a) $k$ is almost integral over $D$,

(b) $D[k][[x]] \subseteq Q(D[[x]])$,

(c) $E[k][[x]] \subseteq Q(D[[x]])$, where $E$ denotes the subring of $D$ generated by the identity element of $D$.

Then (a) implies (b) and (b) implies (c), and when D is root closed (in K) (c) implies (a), i.e., all three conditions are equivalent.

REMARK. Because of a paucity of good examples of non-root-closed domains, we are unable to ascertain whether the assumption of root closure is actually needed in this lemma and in certain later results. However, since the domains in which we are most interested are all root closed (in fact, integrally closed, especially c.i.c. and/or Prüfer), this deficiency does not affect our later theorems.

Before beginning the proof of Lemma 3.1, we need the following lemma.

3.2. Lemma. Suppose $p$ is a power series in $K[[x]]$ which is in $Q(D[[x]])$. Then there is an $a \in D^{*}$ such that $p \in a^{-1}(D[[x / a]])$. In other words, we may rewrite $p$ as $d_{0} / a+\left(d_{1} / a^{2}\right) x+\left(d_{2} / a^{3}\right) x^{2}+\cdots$ where the $d_{i}$ are all in $D$.

Proof. This result is proved computationally by $\mathrm{Ohm}\left[\mathrm{O}_{1}\right.$, Proposition 2.5, p. 327] but for the sake of completeness we present a sketch of a more conceptual proof below. We let $\boldsymbol{p}=\boldsymbol{c} / \boldsymbol{b}$, where $\boldsymbol{c}=c_{0}+c_{1} x+c_{2} x^{2}+\cdots$ and $\boldsymbol{b}=b_{0}+b_{1} x+b_{2} x^{2}$ $+\cdots$. We consider only the case where $b_{0} \neq 0$, since otherwise we can divide out powers of $x$ from numerator and denominator until $b$ has nonzero constant term. Then $b_{0}^{-1} b$ is a power series in $D\left[\left[x / b_{0}\right]\right]$ which has a unit as constant term. Hence its inverse, $\left(b_{0}^{-1} b\right)^{-1}$, which equals $b_{0} b^{-1}$, is also in $D\left[\left[x / b_{0}\right]\right]$ and so is $c\left(b_{0} b^{-1}\right)$. But $\boldsymbol{p}=b_{0}^{-1}\left[\boldsymbol{c}\left(b_{0} \boldsymbol{b}^{-1}\right)\right]$ which shows that $\boldsymbol{p} \in b_{0}^{-1}\left(D\left[\left[x / b_{0}\right]\right]\right)$, and thereby completes the proof.

Proof of 3.1. (a) implies (b). Suppose $k$ is almost integral over $D$. Then there exists a $d \in D^{*}$ such that $d(D[k]) \subseteq D$. Hence for any power series $p$ with coefficients in $D[k], d \boldsymbol{p} \in D[[x]]$. Hence $\boldsymbol{p} \in(D[[x]])_{D^{*}}$ and thus it necessarily lies in $Q(D[[x]])$.

(b) implies (c). Since $E \subseteq D, E[k] \subseteq D[k]$, and similarly for their power series rings.

(c) implies (a). Assume $D$ is root closed, and assume (c) holds. Then the power series $\sum_{n=1}^{\infty} k^{(n+1)^{2}} x^{n}$, which is in $E[k][[x]]$, is also in $Q(D[[x]])$. Hence, by Lemma 3.2 , it lies in $a^{-1}(D[[x / a]])$ for some $a \in D^{*}$. This is the same as saying that for each $n, k^{(n+1)^{2}}=d_{n} / a^{n+1}$ for some $d_{n} \in D$. In other words, $a^{n+1} k^{(n+1)^{2}}$ is in $D$ for all $n$, or $\left(a k^{n+1}\right)^{n+1} \in D$ for all $n$. Since $D$ is root closed and $a k^{n+1} \in K$, we conclude that $a k^{n+1} \in D$ for all $n$, which means $k$ is almost integral over $D$. This completes the proof of Lemma 3.1. 
The relation between quotient fields of power series rings and complete integral closure is further amplified by the proposition that follows next.

3.3. Proposition. Suppose $D_{1}$ and $D_{2}$ are root closed and

$$
Q\left(D_{1}[[x]]\right)=Q\left(D_{2}[[x]]\right) .
$$

Then $\operatorname{CIC}\left(D_{1}\right)=\operatorname{CIC}\left(D_{2}\right)$.

Proof. Assume the hypotheses are satisfied and pick $k \in \operatorname{CIC}\left(D_{1}\right)$. By 3.1, $E[k][[x]] \subseteq Q\left(D_{1}[[x]]\right)$ which equals $Q\left(D_{2}[[x]]\right)$. Hence, again by 3.1 , since $D_{2}$ is root closed $k$ is almost integral over $D_{2}$. By symmetry, $\operatorname{CIC}\left(D_{1}\right)=\operatorname{CIC}\left(D_{2}\right)$.

REMARK. The converse to this theorem is false; that is, there exist root-closed domains which share the same complete integral closure but for which the respective power series rings have different quotient fields. Some examples are presented in detail in the following section.

We are now ready to prove the first of the principal results of this section.

3.4. TheOREM. For a domain $D$, the following properties are equivalent:

(i) $Q(D[[x]]) \neq Q\left(D_{1}[[x]]\right)$ for every overring $D_{1}$ properly containing $D$.

(ii) $D$ is completely integrally closed.

Proof. Assume $D$ is c.i.c. Pick an overring $D_{1} \neq D$, and assume $Q(D[[x]])$ $=Q\left(D_{1}[[x]]\right)$. Pick $k \in D_{1}-D$. Since $D[k]$ lies between $D$ and $D_{1}$, we see that $Q(D[[x]])=Q(D[k][[x]])$. Hence $D[k][[x]]$ is contained in $Q(D[[x]])$. But $D$ is necessarily root closed since it is c.i.c., and hence by $3.1, k$ is almost integral over $D$. But then $k \in D$. This contradicts our choice of $k$ and proves that $D_{1}[[x]]$ cannot have the same quotient field as $D[[x]]$.

Conversely, assume $D$ is not c.i.c. Then there is an element $k \in K-D$ which is almost integral over $D$. So $D[k][[x]]$ is contained in $Q(D[[x]])$, and so is its quotient field. Since the reverse containment of the quotient fields is trivial, the two quotient fields are equal. Q.E.D.

As a slight variation of this last result, we obtain the second principal result of the section, containing the new characterization of one-dimensional Prüfer domains mentioned earlier.

3.5. THEOREM. The following conditions on a domain $D$ are equivalent:

(i) For any two overrings $D_{1} \neq D_{2}$ of $D, Q\left(D_{1}[[x]]\right) \neq Q\left(D_{2}[[x]]\right)$.

(ii) $D$ and all its overrings are completely integrally closed.

(iii) $D$ is Prüfer with Krull dimension one.

Proof. (i) implies (ii). This is just a special case of (i) implies (ii) in the previous theorem.

(ii) implies (i). Pick any two overrings $D_{1}$ and $D_{2}$ such that $Q\left(D_{1}[[x]]\right)=$ $Q\left(D_{2}[[x]]\right)$. Since they are both c.i.c., we see that $D_{1}=\operatorname{CIC}\left(D_{1}\right)=\operatorname{CIC}\left(D_{2}\right)=D_{2}$, the middle equality coming from 3.3. So $D_{1}$ and $D_{2}$ are not distinct, and we are done. 
(ii) implies (iii). It is well known that any domain for which all overrings are integrally closed is Prüfer $\left[\mathrm{G}_{1}\right.$, Theorem 22.2, p. 309]. Moreover, a valuation ring is c.i.c. iff it is rank one $\left[G_{1}\right.$, Theorem 14.5, p. 179]. Hence any domain satisfying (ii) is Prüfer, with only rank-one valuation rings among its valuation overrings. Hence it must also have Krull dimension one $\left[G_{1}\right.$, p. 348].

(iii) implies (ii). Suppose $D$ is Prüfer. Then any overring (including $D$ ) is an intersection of localizations of $D$ at prime ideals [D, Proposition 2, p. 197]. But these localizations are necessarily valuation rings. Moreover, by the correspondence between the chains of those prime ideals of $D$ lying inside $P$ and the chains of primes of $D_{P}$, whenever $D$ is one dimensional so is $D_{P}$. Hence the valuation rings $D_{P}$ are all of rank one, hence c.i.c. Thus any overring of $D$ is an intersection of c.i.c. rings, and consequently it must be c.i.c. as well. This completes the proof of Theorem 3.5.

At this point we would like to restrict our attention to the quotient overrings of $D$ and to the analogs to Theorems 3.4 and 3.5 in this new context. First we need some new terminology.

3.6. Definitions. An element $a$ of $D$ is said to be bounded if $\bigcap_{i=1}^{\infty} a^{i} D \neq 0$. In particular, every unit of $D$ is always bounded. If every nonunit of $D$ is unbounded, then we say $D$ is Archimedean (denoted Arch.). (The use of the word "Archimedean" is motivated by the strong parallel from the theory of partially-ordered groups. See, for example, [F, p. 12].)

One of the nicer aspects of considering only quotient rings is that we can avoid the necessity of assuming root closure of our domains. The following lemma illustrates this new-found freedom.

3.7. Lemma. In Lemma 3.1, if $k$ is of the form $a^{-1}$ for some a in $D^{*}$, then (c) implies (a) without any restriction on the domain $D$.

Proof. First we observe that $a$ is bounded in $D$ iff $a^{-1}$ is almost integral over $D$, simply because $d \in a^{n} D$ iff $d\left(a^{-1}\right)^{n} \in D$. Now suppose $E\left[a^{-1}\right][[x]]$ is contained in $Q(D[[x]])$. Then $E[[x / a]]$, which is contained in $E\left[a^{-1}\right][[x]]$, is also a subset of $Q(D[[x]])$. In particular, the power series $\sum_{j \in J}(x / a)^{j}$ is in $Q(D[[x]])$ for any set $J$ of nonnegative integers. But the proof of Theorem 2.1 showed that if $a$ is unbounded, then certain power series of the type above were transcendental over $Q(D[[x]])$. Hence $a$ is bounded, and $a^{-1}$ is almost integral over $D$. End of proof.

Now we are ready to state our first result concerning the relationship between the quotient fields of $D[[x]]$ and $D_{S}[[x]]$. Again note the absence of any assumption related to root closure.

3.8. THEOREM. Let $S$ be a multiplicative subset of $D^{*}$. Then the following statements are equivalent:

(i) $Q\left(D_{S}[[x]]\right)=Q(D[[x]])$.

(ii) $D_{S}[[x]] \subseteq(D[[x]])_{D^{*}}$.

(iii) For every sequence $s_{1}, s_{2}, s_{3}, \ldots$ of elements of $S, \bigcap_{i=1}^{\infty} s_{i} D \neq 0$. 
This is merely a restatement of Gilmer's result $\left[\mathrm{G}_{3}\right.$, Theorem 1] in a more general setting, with $S$ replacing $D^{*}$ and $D_{S}$ replacing $K$. It is easy to see that (iii) implies (ii) and that (ii) implies (i). The proof that (i) implies (iii) utilizes the same calculations as those Gilmer used, and we will omit them here.

3.9. CoROllaRY. D has the property that $Q(D[[x]]) \neq Q\left(D_{S}[[x]]\right)$ for every proper quotient overring $D_{S}$ of $D$ iff $D$ is Archimedean.

3.10. COROLlaRY. D has the property that for any two distinct quotient overrings $D_{S}$ and $D_{T}, Q\left(D_{S}[[x]]\right) \neq Q\left(D_{T}[[x]]\right)$ iff $D$ and all its quotient rings are Archimedean.

Proof of 3.9. Suppose $Q\left(D_{S}[[x]]\right)=Q(D[[x]])$ for some multiplicative subset $S$ of $D^{*}$, which is not contained in the set of units of $D$. Then we may apply part (iii) of 3.8 to the set $a, a^{2}, \ldots$ in $S$, for any nonunit $a$ in $S$, and conclude $\bigcap a^{i} D \neq 0$. Therefore $D$ is not Arch.

Conversely, if $D$ is not Arch. and $a$ is a nonunit satisfying $\cap a^{i} D \neq 0$, then the set $S=\left\{a, a^{2}, a^{3}, \ldots\right\}$ clearly will satisfy $Q\left(D_{S}[[x]]\right)=Q(D[[x]])$.

Proof of 3.10. We need the fact that a quotient overring of $D_{S}$ is also a quotient overring of $D$ [G-O, Proposition 1.3, p. 98]. Thus if for any two distinct quotient rings, $Q\left(D_{T}[[x]]\right) \neq Q\left(D_{S}[[x]]\right)$, then by 3.9 each $D_{S}$ is necessarily Arch.

Conversely, suppose for some pair $D_{S} \neq D_{T}, Q\left(D_{S}[[x]]\right)=Q\left(D_{T}[[x]]\right)$. Assume without loss of generality that $D_{S} \ddagger D_{T}$. Since $D \subseteq D_{T}$, there must be an $s \in S$ such that $s^{-1} \notin D_{T}$. But

Hence

$$
E\left[s^{-1}\right] \subseteq D\left[s^{-1}\right] \subseteq D_{s}
$$

$$
E\left[s^{-1}\right][[x]] \subseteq D_{S}[[x]] \subseteq Q\left(D_{T}[[x]]\right) .
$$

But applying Lemma 3.7 to $D_{T}$, we see that $s^{-1}$ is almost integral over $D_{T}$, which means $s$ is bounded in $D_{T}$. However, $s^{-1}$ is not in $D_{T}$ by our choice of $s$, so $s$ is not a unit of $D_{T}$. Since $D_{T}$ has a nonunit which is bounded, it is not Arch. which is what we wanted to prove.

REMARK. It would be highly desirable to have a concrete characterization of the class of domains satisfying the conditions of 3.10 , but none is available at the present time. Several things are clear, however.

Every one-dimensional domain satisfies 3.10. (Apply $\left[\mathrm{O}_{1}\right.$, Corollary 1.4 , p. 323]. This reference shows that any $D$ for which every nonunit is in a minimal prime is Archimedean.) For Prüfer domains, the converse is also valid since a valuation overring is Archimedean iff it is c.i.c. and hence of rank one. Thus the Prüfer domains satisfying 3.10 are exactly the one-dimensional ones.

However, this class of domains contains more than just one-dimensional Prüfer domains. For example, every quotient ring of a Krull domain is again a Krull domain [B, Proposition 6, p. 10]. Hence every quotient ring of a Krull domain is c.i.c. and therefore Arch. Therefore, all Krull domains satisfy the conditions of 3.10 . 
4. Some examples and their relation to the group of divisibility. Proposition 3.3 shows that, for root-closed domains $D_{1}$ and $D_{2}$, equality of the quotient fields of $D_{1}[[x]]$ and $D_{2}[[x]]$ implies the equality of the complete integral closures of $D_{1}$ and $D_{2}$. This result raises the following question: To what extent does the complete integral closure of a root-closed domain $D$ determine the quotient field of $D[[x]]$ ? Our first partial result in this direction is a positive one, namely that, for a valuation ring $D, \mathrm{CIC}(D)$ completely determines $Q(D[[x]])$ whenever CIC $(D) \neq K$. Attempts to pursue this question further, however, yield negative answers on two different fronts. First we show in Example 4.7 that the assumption CIC $(D) \neq K$ may not be omitted from the valuation rings being considered. In particular, two valuation rings in the same quotient field $K$ with both complete integral closures equal to all of $K$ may have different quotient fields for their power series rings. Secondly, even among those Bezoutian domains $D$ with $\mathrm{CIC}(D) \neq K$, the complete integral closure fails to determine $Q(D[[x]])$, as Example 4.8 shows.

We begin with the positive result mentioned above.

4.1. Proposition. Let $U$ and $V$ be valuation rings in the same quotient field $K$, and assume $\mathrm{CIC}(U)=\mathrm{CIC}(V)=W \neq K$. Then

$$
Q(U[[x]])=Q(V[[x]])=Q(W[[x]]) .
$$

In other words, $\mathrm{CIC}(V)$ determines $Q(V[[x]])$, and in particular, for $Q(V[[x]])$ we could always take the quotient field of $\mathrm{CIC}(V)[[x]]$.

The proof of this proposition will be an elementary application of the following proposition, which has intrinsic value for two reasons. First, it provides a ready source of domains for which $Q\left(D_{1}[[x]]\right)=Q\left(D_{2}[[x]]\right)$. Second, it reiterates the strong parallel between complete integral closures and quotient fields of power series rings. (Compare it with the statement [G-H, Lemma 5, p. 358] that if $D_{1} \subseteq D_{2}$ and the conductor of $D_{1}$ in $D_{2}$ is nonzero, then $\operatorname{CIC}\left(D_{1}\right)=\operatorname{CIC}\left(D_{2}\right)$.)

First we need some terminology. The conductor of $D_{1}$ in $D_{2}$, where $D_{1}$ is contained in $D_{2}$, is the set of all elements $z \in D_{1}$ such that $z D_{2} \subseteq D_{1}$. It is also characterized as the largest ideal of $D_{1}$ which is also an ideal of $D_{2}$. The conductor of $D_{1}$ in $D_{2}$ is nonzero iff $D_{2}$ is contained in a principal $D_{1}$-submodule of $K$. For example, $y$ is almost integral over $D$ iff the conductor of $D$ in $D[y]$ is nonzero [G-H, Lemma 4, p. 358].

4.2. Proposition. If $D_{1}$ is contained in $D_{2}$ and the conductor of $D_{1}$ in $D_{2}$ is nonzero, then $Q\left(D_{1}[[x]]\right)=Q\left(D_{2}[[x]]\right)$.

Proof of 4.2. If the conductor of $D_{1}$ in $D_{2}$ is nonzero, then there is a $d \in D_{1}^{*}$ such that $d D_{2} \subseteq D_{1}$. Hence if $p$ is any power series in $D_{2}[[x]]$, then clearly $d p \in D_{1}[[x]]$. So $\boldsymbol{p} \in\left(D_{1}[[x]]\right)_{D_{1}^{*}}$, which we know is contained in $Q\left(D_{1}[[x]]\right)$, and therefore $D_{2}[[x]] \subseteq Q\left(D_{1}[[x]]\right)$. The equality of the two quotient fields is now a triviality. 
RemarK. The converse to this proposition is false, i.e. there exist $D_{1} \subset D_{2}$ such that $Q\left(D_{1}[[x]]\right)=Q\left(D_{2}[[x]]\right)$ and the conductor of $D_{1}$ in $D_{2}$ is zero. For example, any domain $D$ which is not a field but for which $Q(D[[x]])=Q(K[[x]])$ will provide a counterexample, since the conductor of $D$ in $K$ is always zero. $\left(\right.$ See $\left[\mathrm{G}_{3}\right]$ for such a domain $D$.) Other examples may also be constructed utilizing the techniques in Example 4.8 together with Theorem 3.8.

Proof of 4.1. By symmetry it will suffice to show $Q(V[[x]])=Q(W[[x]])$, and by 4.2 it will suffice to show that the conductor of $V$ in $W$ is nonzero. Since $V$ is a valuation ring and $W$ an overring, $V$ has a prime ideal $P$ such that $W=V_{P}\left[G_{1}\right.$, Theorem 14.6, p. 181]. Moreover, the ideal $P$ is also an ideal of $W$, being equal to $P \cdot W\left[\mathrm{G}_{1}\right.$, Theorem 14.6, part (b), p. 182]. Finally, since $W$ is not equal to $K, P$ is not equal to zero. So there is a nonzero ideal of $V$ which is also an ideal of $W$. Since the conductor of $V$ in $W$ is the largest such ideal, it must be nonzero as well. Q.E.D.

ReMARKS. (1) Note that in the proof of Proposition 4.1 we never used the fact that $W$ was the complete integral closure of $V$, but just that it was a proper overring of $V$. (In this sense, "proper" means only that it is unequal to $K$.) This observation is not surprising since for two valuation rings with proper complete integral closures, sharing some common proper overring is equivalent to having the same complete integral closures. (Apply the fact that the complete integral closure in such cases is always the unique maximal proper overring of $V$.) Moreover, we also never really used the assumption that $\mathrm{CIC}(V) \neq K$, except to find a common proper overring. Thus we could apply this same argument to valuation rings with complete integral closures equal to all of $K$, for example, and prove that the rings of the type in Example 4.7, with different quotient fields for their power series rings, cannot possibly share a common proper overring.

(2) Proposition 4.1 suggests that, since $\mathrm{CIC}(V)[[x]]$ and $V[[x]]$ share the same quotient field when $\mathrm{CIC}(V) \neq K$, the fact that $\mathrm{CIC}(V)$ is completely integrally closed must play an important role in these considerations. (By Proposition 3.3, CIC $(V)$ has to be c.i.c. for the quotient fields to be equal.) This does not appear to be the case for Bezoutian domains, however, since the one we will construct in Example 4.8 also has the property that its complete integral closure is completely integrally closed and unequal to $K$, but the power series rings $D[[x]]$ and $\mathrm{CIC}(D)[[x]]$ fail to share the same quotient fields.

The remainder of this section will be devoted to examples which provide negative answers to our attempts to extend the preceding result. In the construction of our examples we will make extensive use of the concepts of semivaluations and divisibility groups. This approach is a natural outgrowth of the consideration of the case of valuation rings, in which it is easily seen that many of the properties of interest here are properties of their value groups, and independent of numbertheoretic, congruence, or other properties of the ring. For example, Gilmer $\left[\mathrm{G}_{3}\right]$ constructs an example of a valuation ring $D$ for which $Q(D[[x]])=Q(K[[x]])$ by 
first constructing the value group needed and then citing the existence of a valuation ring with the specified group as its value group. The proper generalizations of valuation and value group to arbitrary domains are the semivaluation and the divisibility group, respectively, and we define them next.

4.3. Definitions. The divisibility group of the domain $D$ is the partially-ordered abelian group of all nonzero principal fractional ideals of $D$, with $k_{1} D \geqq k_{2} D$ iff $k_{1} D \subseteq k_{2} D$. In this group note that the principal ideal generated by 1 , i.e. all of $D$, is the identity element (in additive notation, the zero element). Hence the set of "positive" elements, i.e. those $\geqq 0$, is precisely the set of nonzero principal ordinary ideals, i.e. principal ideals of $D$. Note that a divisibility group always has the property of being filtered (also called directed [F, p. 10]); that is, every set of two elements has an upper bound. This follows from the observation that the ideal $a_{1} a_{2} D$ is an upper bound for the set $\left\{\left(a_{1} / b_{1}\right) D,\left(a_{2} / b_{2}\right) D\right\}$ whenever $a_{1} ; a_{2}, b_{1}, b_{2}$ are nonzero elements of $D$.

A semivaluation of $K\left[\mathrm{O}_{2}, \S 1\right]$ is a map $w$ of $K^{*}$ into a partially-ordered abelian group $G$ satisfying

(I) $w(a b)=w(a)+w(b)$,

(II) $w(-1)=0$,

(III) for all $a, b \in K^{*}$ with $a \neq-b$, and all $g \in G$, if $w(a) \geqq g$ and $w(b) \geqq g$, then $w(a+b) \geqq g$.

The map from $K^{*}$ onto the divisibility group of $D$ taking $k$ to $k D$ will always be a semivaluation of $K$. (Note that the kernel of this map is $U$, the group of units of $D$, and hence the divisibility group is often defined equivalently to be $K^{*} / U$ with the appropriate order.) Conversely, given any semivaluation $w$ of $K$ onto a filtered group $G$, the set $\{0\} \cup\left\{k \in K^{*} \mid w(k) \geqq 0\right\}$, called the semivaluation ring of $w$, is a domain with quotient field $K$ and divisibility group order-isomorphic to $G$. (In case $G$ is not filtered, the semivaluation ring will still be a subring of $K$, but its quotient field will be properly less than $K$.) As suggested earlier, every valuation is a semivaluation, and for a valuation ring, the divisibility group and the value group are the same.

As we mentioned briefly for valuation rings, many of the properties of domains which interest us here are really properties of their divisibility groups, in the sense that any two domains with the same divisibility groups will necessarily share the given property. To illustrate this point, we let $D$ be a domain with divisibility group $G$ and we let $w$ be the associated semivaluation. First, if $w(d)=g$, then $w\left(d^{n}\right)=n g$. Hence $d$ is bounded in $D$ iff the set $\{g, 2 g, 3 g, \ldots\}$ has an upper bound in $G$. (It will be convenient to call such an element $g$ a bounded element of $G$, for obvious reasons.) In general, if $\left\{d_{1}, d_{2}, \ldots\right\}$ is a subset of $D^{*}$, then $d \in \bigcap_{i=1}^{\infty} d_{i} D$ iff $w(d)$ is an upper bound for the set $\left\{w\left(d_{1}\right), w\left(d_{2}\right), \ldots\right\}$. Thus such properties as being root closed, being Archimedean, or having $Q(D[[x]])=Q(K[[x]])$ all will depend only on the divisibility group having the appropriate corresponding property. 
There are somewhat more subtle examples of domain properties that really belong to the divisibility group, and we shall need two of them in a later example. First, observe that from our earlier definition of boundedness in $D$, it is easy to see that the set $B$ of all bounded elements of $D$ is a multiplicative subset of $D^{*}$. Thus it is natural to form the quotient ring $D_{B}$. Since $D$ is Archimedean iff $D=D_{B}$, we shall call $D_{B}$ the Archimedean closure of $D$, and denote it AC (D). Despite the fact that $\mathrm{AC}(D)$ is formed by making all the bounded elements of $D$ invertible, it is not true that all the remaining nonunits of $\mathrm{AC}(D)$ are unbounded. In other words, the Archimedean closure of $D$ is not always Archimedean. The parallel to the case of the complete integral closure not necessarily being c.i.c. is more than just a coincidence, as the following proposition shows. Specifically, for a large class of domains, the concepts of complete integral closure and Archimedean closure are identical, and hence the existing examples of Bezoutian domains for which $\mathrm{CIC}(D)$ is not c.i.c. also have the property that $\mathrm{AC}(D)$ is not $\operatorname{Arch}$. For instance, see Heinzer's example $[\mathrm{H}]$.

4.4. Proposition. Let $D$ be a domain with the QR-property. (I.e., every overring is a quotient ring. E.g., any Bezoutian domain. See [G-O] or $\left[\mathrm{G}_{1}, \S 23\right]$.) Then $\mathrm{CIC}(D)=\mathrm{AC}(D)$. Hence $D$ is completely integrally closed iff $D$ is Archimedean.

Proof. First recall our observation in the proof of 3.7 that $a^{-1}$ is almost integral over $D$ iff $a$ is bounded in $D$. Since AC $(D)$ is generated by $D$ and the inverses of the bounded elements of $D$ and since these inverses are all almost integral over $D$, it follows that $\mathrm{AC}(D)$ is always contained in CIC $(D)$ without any conditions on $D$. Conversely, if $D$ has the QR-property, then CIC $(D)$ is a quotient ring of $D$. Now it is elementary to verify that if $D^{\prime}$ is any quotient overring of $D$, it may be considered as a quotient with respect to the multiplicative system obtained by intersecting $D$ with the set of units of $D^{\prime}$. In our special case, CIC $(D)$ is a quotient ring with respect to the set of all elements of $D^{*}$ which are invertible in CIC (D). This is precisely the set of all $s$ in $D^{*}$ such that $s^{-1}$ is almost integral over $D$, which we know to be the set $B$ of all bounded elements. Thus $\mathrm{CIC}(D)=D_{B}=\mathrm{AC}(D)$. The final sentence, which is a restatement of Proposition 23.6 in $\left[G_{1}\right]$, now follows immediately. Q.E.D.

Now since $\mathrm{AC}(D)$ is obtained by forming the quotient ring with respect to the set of bounded elements of $D$, and since boundedness is a property that can be stated in terms of the divisibility group, it seems reasonable to think that many properties of $\mathrm{AC}(D)$ are dependent only on the divisibility group of $D$. The two properties which we will need in Example 4.8 fit into this category, as the following proposition demonstrates.

4.5. Proposition. Let $D$ be a domain with divisibility group $G$. Let $w$ be the associated semivaluation of $K^{*}$ onto $G$, and let $G_{+}$denote the set of positive elements of $G$ (including zero). Then 
(a) $D[[x]]$ and $\mathrm{AC}(D)[[x]]$ have the same quotient field iff every countable sequence of bounded elements of $G_{+}$has an upper bound.

(b) $\mathrm{AC}(D)$ is not Archimedean iff the following set of elements exists in $G_{+}$: an unbounded element $g$, a countable sequence of bounded elements $h_{1}, h_{2}, \ldots$, and an additional element $h$ (bounded or unbounded), which together satisfy

$$
h+h_{n} \geqq n g \text { for all } n \text {. }
$$

Proof. (a) Let $B$ be the set of bounded elements, and recall that $\mathrm{AC}(D)=D_{B}$. By Theorem 3.8, $D[[x]]$ and $D_{B}[[x]]$ share the same quotient field iff $\cap b_{i} D \neq 0$ for every sequence $b_{1}, b_{2}, \ldots$ of elements of $B$. This is clearly equivalent to every sequence of bounded positive elements of $G$ having an upper bound.

(b) Suppose $D_{B}$ is not Arch. Then there exists a nonunit, say $\mathrm{ra}^{-1}(r \in D, a \in B)$ of $D_{B}$ which is bounded in $D_{B}$. Since $r a^{-1}$ is a nonunit of $D_{B}$, so is $r$, which means $r$ is unbounded in $D$. The fact that $r a^{-1}$ is bounded in $D_{B}$ implies that there is a nonzero element, say $s b^{-1}(s \in D, b \in B)$, in $\bigcap_{i=1}^{\infty}\left(r a^{-1}\right)^{i} D_{B}$. Hence, for each $n$ there is a $t_{n}$ in $D$ and a $c_{n}$ in $B$ such that

Cross-multiplying yields

$$
s b^{-1}=\left(r a^{-1}\right)^{n} t_{n} c_{n}^{-1} .
$$

which implies

$$
s a^{n} c_{n}=r^{n} t_{n} b
$$

$$
s a^{n} c_{n} \in r^{n} D \text { for each } n .
$$

If we let $w(r)=g, w(s)=h$, and $w\left(a^{n} c_{n}\right)=h_{n}$, then it can be seen that $g$ is unbounded and each $h_{n}$ is bounded. The latter observation follows from the fact that $a$ and each $c_{n}$ are in $B$ and $B$ is closed under multiplication. Applying $w$ to both sides of (4.6.2) then yields (4.6.1), which is what we wanted to prove.

Now suppose there exist $g, h$, and $h_{1}, h_{2}, \ldots$ as in the proposition. Let $r, s$, and $b_{1}, b_{2}, \ldots$ be chosen in $D$ to satisfy $w(r)=g, w(s)=h$, and $w\left(b_{n}\right)=h_{n}$ for each $n$. Then clearly $r$ is unbounded in $D$, hence a nonunit of $\mathrm{AC}(D)$. But (4.6.1) implies that $s b_{n}$ is in $r^{n} D$ for each $n$. Hence $s$ is in $r^{n} D_{B}$ for each $n$, and $s$ is nonzero, which proves $D_{B}$ is not Arch. Q.E.D.

REMARK. It should be noted that the property of being completely integrally closed and even the property of the complete integral closure being completely integrally closed are also properties of the divisibility group, but we will have no need for them here. For instance, Heinzer's demonstration $[\mathrm{H}, \S 2]$ of a domain with complete integral closure which is not completely integrally closed shows that any domain with the same divisibility group will share this property.

All of this discussion of properties of the divisibility group would be useless in constructing examples of domains without a theorem on the existence of domains with certain specified divisibility groups. The theorem we need is due to Jaffard [J, Theorem 3, p. 78] and $\mathrm{Ohm}\left[\mathrm{O}_{1}\right.$, p. 329] and, while restricted only to latticeordered groups, is very useful because of the abundance and ease of construction of such groups. 
THEOREM (WITHOUT PROOF). Given a lattice-ordered group $G$, there is a Bezoutian domain $D$ with divisibility group $G$.

While there are several standard constructions of valuation rings with specified totally-ordered groups as their divisibility groups, none of them shows immediately how to imbed two valuation rings with two specified value groups into the same quotient field. Since we will need such an imbedding theorem in Example 4.7, and since it is an elementary consequence of Jaffard's theorem, we state and prove it now. It is stated below in far more general terms than we need, simply because the proof appears to be no more difficult.

4.6. THEOREM. Let $\left\{L_{\alpha} \mid \alpha \in A\right\}$ be a collection of lattice-ordered groups. Then there is a field $K$ which is simultaneously the quotient field of each one of a collection of domains $\left\{D_{\alpha} \mid \alpha \in A\right\}$ with the property that, for each $\alpha$, the divisibility group of $D_{\alpha}$ is $L_{\alpha}$.

Proof. Let $G$ be the weak direct sum of the groups $L_{\alpha}$ with the product ordering. It is well known that $G$ is also a lattice-ordered group [F, p. 23], and therefore we may construct a domain $D$ with divisibility group $G$. Let $K$ denote the quotient field of $D$ and let $w$ denote the semivaluation from $K^{*}$ onto $G$ which corresponds to $D$. Let $\pi_{\alpha}$ denote the projection map from $G$ onto $L_{\alpha}$. Then it is routine to prove that the composite map $\pi_{\alpha} \circ w$ is a semivaluation of $K^{*}$ onto $L_{\alpha}$. Properties (I) and (II) follow from the fact that $\pi_{\alpha}$ is a group homomorphism, and (III) comes from the definition of projection map and product ordering. Hence the semivaluation ring $D_{\alpha}$ of $\pi_{\alpha} \circ w$ has quotient field $K$ and divisibility group $L_{\alpha}$. Q.E.D.

Now we are ready to present the examples we need to show the limitations on when CIC $(D)$ determines $Q(D[[x]])$.

4.7. ExAmple. We will construct a pair of valuation rings, $V$ and $W$, in the same quotient field $K$ satisfying

(A) $\operatorname{CIC}(V)=\mathrm{CIC}(W)=K$, and

(B) $Q(V[[x]]) \neq Q(W[[x]])$.

Specifically, we will show

(B') $Q(V[[x]]) \neq Q(K[[x]])$, and

(B") $Q(W[[x]])=Q(K[[x]])$.

This pair will then illustrate the fact that in case a valuation ring $D$ has complete integral closure equal to its quotient field, then $\operatorname{CIC}(D)$ fails to determine $Q(D[[x]])$.

We begin by constructing the value groups $G$ and $H$ for $V$ and $W$ respectively. For the group $G$ we take a weak direct sum of a countable number of copies of the integers, indexed by the positive integers, and order it with a reverse lexicographic ordering. In other words, $(1,0,0, \ldots)$ is less than $(0,1,0, \ldots)$ and so forth; since we use the weak direct sum, every element has a last nonzero entry at which to begin comparing it with any other element. Let $V$ be any valuation ring with $G$ as its value group. 
First we want to show CIC $(V)=K$. Again we cite the fact that for any domain $D$ and element $a \in D^{*}, a^{-1}$ is almost integral over $D$ iff $a$ is bounded in $D$. Thus we can see that CIC $(D)=K$ iff every $a \in D^{*}$ is bounded in $D$, which will be true iff every positive element $g$ in the divisibility group of $D$ is bounded (i.e., the set $\{g, 2 g, 3 g, \ldots\}$ has an upper bound). So to show $\operatorname{CIC}(V)=K$, we need only show that every $g$ in $G_{+}$is bounded. Pick any $g$ in $G_{+}$, and suppose it has all of its nonzero entries within the first $n$ coordinates. Hence $2 g, 3 g, \ldots$ also have all of their nonzero entries within the first $n$ coordinates, which means that any positive element of $G$ with a nonzero entry after the $n$th position is an upper bound for the set $\{g, 2 g, 3 g, \ldots\}$. Hence every $g$ in $G_{+}$is bounded and therefore CIC $(V)=K$.

To see that $Q(V[[x]]) \neq Q(K[[x]])$, by Gilmer's characterization $\left[\mathrm{G}_{3}\right]$ we need only show that, for some sequence of nonzero elements $a_{1}, a_{2}, \ldots$ of $V, \cap a_{i} V=0$. But this means we need only a countable sequence of elements of $G_{+}$without an upper bound. The sequence $g_{1}=(1,0,0, \ldots), g_{2}=(0,1,0, \ldots), \ldots$ clearly suffices.

For the value group $H$, we will take the same group Gilmer used to construct a valuation ring satisfying $Q(D[[x]])=Q(K[[x]])\left[\mathrm{G}_{3}\right]$. This group-a reverselexicographically-ordered weak direct sum of a best-well-ordered uncountable set of copies of the integers-is basically a much larger version of the group $G$ just constructed. It is a triviality that every positive element of $H$ is bounded and hence that any valuation ring $W$ with $H$ as its value group must satisfy $\mathrm{CIC}(W)=K$. And of course, our choice of $H$ guarantees $Q(W[[x]])=Q(K[[x]])$.

All that remains to complete Example 4.7 is to show that two such valuation rings $V$ and $W$ with $G$ and $H$ as value groups can be imbedded in the same quotient field. But this is an immediate consequence of Theorem 4.6. Q.E.D.

4.8. ExAmple. Our final example will show that Proposition 4.1 cannot be extended from valuation rings to Bezoutian domains, or even to the subclass of all those Bezoutian domains whose complete integral closure is completely integrally closed. To show that CIC $(D)$ does not determine $Q(D[[x]])$ in the case of Bezoutian domains with proper complete integral closures, we will construct a Bezoutian domain $D$ which shares with its Archimedean closure $D_{B}$ (also a Bezoutian domain, see [J, Theorem 2, p. 95]) the same proper complete integral closure but not the same quotient field for its power series ring. The precise conditions that we need on $D$ are the following:

(A) $\mathrm{CIC}(D) \neq K$.

(B) $Q\left(D_{B}[[x]]\right) \neq Q(D[[x]])$.

(C) $\operatorname{CIC}\left(D_{B}\right)=\operatorname{CIC}(D)$.

Since both $D_{B}$ and $D$ are Bezoutian, then $D_{B}=\mathrm{CIC}(D)$ by Proposition 4.4. Hence (C) may be replaced by

$\left(C^{\prime}\right) \operatorname{CIC}\left(D_{B}\right)=D_{B}$, i.e. $D_{B}$ is completely integrally closed, which may in turn be replaced, using the same argument, by

(C') $D_{B}$ is Archimedean. 
All that remains is to construct a Bezoutian domain $D$ with properties (A), (B), and $\left(C^{\prime \prime}\right)$ above. But these three conditions are all conditions on the divisibility group of $D$ alone. Thus we need only find a lattice-ordered group $G$ with the corresponding three properties, and use the Jaffard-Ohm Theorem to prove the existence of the Bezoutian domain $D$ that we need.

To construct $G$, we first let $T$ be the totally-ordered abelian group obtained by lexicographically ordering the direct sum of two copies of the integers. In this ordering, $(1,0)$ is greater than $(0,1)$ and so forth. Let $G$ be the set of all functions from the positive integers into $T$ with finite support, i.e., with nonzero value at only finitely many points. Then it is easy to verify that $G$ is a lattice-ordered group with respect to pointwise addition and pointwise ordering. The set of all positive elements of $G$ is precisely the set of all nonnegative-valued functions, and the bounded positive elements are those which also satisfy $f(n)=\left(0, z_{n}\right)$ for each $n$. This is because if $y$ is a positive integer, there is no upper bound in $T$ for the set $\{(y, z),(2 y, 2 z), \ldots\}$. Hence $G$ has unbounded positive elements and (A) must be satisfied for the corresponding domain $D$.

Next, the set of functions $\left\{f_{1}, f_{2}, \ldots\right\}$ defined by

$$
\begin{aligned}
& f_{i}(n)=(0,0) \text { if } i \neq n, \\
& =(0,1) \text { if } i=n \text {, }
\end{aligned}
$$

is, by the preceding, a countable set of bounded positive elements of $G$. Clearly this set can have no upper bound in $G$, since such an upper bound would have to have an infinite number of nonzero entries. Hence by part (a) of Proposition 4.5, $D$ will necessarily satisfy (B).

Finally, to show $\left(\mathrm{C}^{\prime \prime}\right)$, we suppose $g, h$, and $h_{1}, h_{2}, \ldots$ exist as in part (b) of 4.5 . Then since $g$ is unbounded, it must have some value which is not of the form $(0, z)$. Without loss of generality, assume $g(1)=(1,0)$. Then the assumption that $h+h_{n} \geqq n g$ for all $n$, when applied to the function values at 1 , yields

$$
h(1)+h_{n}(1) \geqq(n, 0) \text { for all } n \text {. }
$$

But the $h_{n}$ are all bounded, which means $h_{n}(1)=\left(0, z_{n}\right)$ for some integer $z_{n}$. Therefore (4.8.1) becomes

$$
h(1) \geqq\left(n,-z_{n}\right) \text { for all } n,
$$

which implies that the first coordinate of $h(1)$ is $\geqq n$ for all $n$. This absurdity proves that $g, h, h_{1}, h_{2}, \ldots$ cannot exist as in 4.6, part (b). Hence $D_{B}$ is Archimedean and the example is complete.

\section{BIBLIOGRAPHY}

[B] N. Bourbaki, Algèbre commutative. Chapitre 7, Hermann, Paris, 1965.

[D] E. D. Davis, Overrings of commutative rings. II. Integrally closed overrings, Trans. Amer. Math. Soc. 110 (1964), 196-212. MR 28 \#111. 
[F] L. Fuchs, Partially ordered algebraic systems, Pergamon Press, New York; AddisonWesley, Reading, Mass., 1963. MR 30 \#2090.

[G $\left.\mathrm{G}_{1}\right]$ R. Gilmer, Multiplicative ideal theory, Queen's Papers in Pure and Appl. Math., no. 12, Queen's University, Kingston, Ontario, 1968. MR 37 \#5198.

$\left[\mathrm{G}_{2}\right] \longrightarrow$, Domains in which valuation ideals are prime powers, Arch. Math. (Basel) 17 (1966), 210-215. MR 37 \#6273.

$\left[\mathrm{G}_{3}\right] \longrightarrow$, A note on the quotient field of the domain $D[[x]]$, Proc. Amer. Math. Soc. 18 (1967), 1138-1140. MR 36 \#155.

[G-H] R. Gilmer and W. Heinzer, On the complete integral closure of an integral domain, J. Austral. Math. Soc. 6 (1966), 351-361. MR 36 \#3766.

[G-O] R. Gilmer and J. Ohm, Integral domains with quotient overrings, Math. Ann. 153 (1964), 97-103. MR 28 \#3051.

[H] W. Heinzer, Some remarks on complete integral closure, J. Austral. Math. Soc. 9 (1969), 310-314.

[J] P. Jaffard, Les systèmes d'idéaux, Travaux et Recherches Mathématiques, IV, Dunod, Paris, 1960. MR 22 \#5628.

$\left[\mathrm{O}_{1}\right] \mathrm{J} . \mathrm{Ohm}$, Some counterexamples related to integral closure in $D[[x]]$, Trans. Amer. Math. Soc. 122 (1966), 321-333. MR 34 \#2613.

$\left[\mathrm{O}_{2}\right]-$, Semi-valuations and groups of divisibility, Canad. J. Math. 21 (1969), 576-591. MR 39 \#4146.

[R] R. Rivet, Sur le corps des fractions d'un anneau de séries formelles à coefficients dans un anneau de valuation discrète, C. R. Acad. Sci. Paris Sér. A-B 264 (1967), A1047-A1049. MR 35 \#4206.

UNIVERSITY OF WISCONSIN, MADISON, WisCONSIN 53706

WisCONSIN STATE UNIVERSITY, WHITEWATER, WISCONSIN 53190 